Helena Hemiko Iwamoto Fernanda Carolina de Camargo ${ }^{2}$ Laureni Conceição Tavares ${ }^{3}$ Sybelle de Souza Castro Miranzi ${ }^{4}$

\section{Acidentes de trabalho fatais e a qualidade das informa- ções de seus registros em Uberaba, em Minas Gerais e no Brasil, 1997 a 2006}

\author{
Fatal occupational injuries and the quality of recorded \\ information for the city of Uberaba, the State of Minas Gerais, \\ and Brazil, between 1997 and 2006
}

${ }^{1}$ Enfermeira. Doutora em Enfermagem Fundamental. Professora do Programa de Pós-Graduação em Atenção à Saúde da Universidade Federal do Triângulo Mineiro (UFTM), Uberaba, MG, Brasil.

${ }^{2}$ Enfermeira. Mestre em Atenção à Saúde. Professora Assistente do Departamento de Medicina Social da Universidade Federal do Triângulo Mineiro (UFTM), Uberaba, MG, Brasil.

${ }^{3}$ Fonoaudióloga. Mestranda em Atenção à Saúde do Programa de Pós-Graduação em Atenção à Saúde da Universidade Federal do Triângulo Mineiro (UFTM), Uberaba, MG, Brasil.

${ }^{4}$ Enfermeira. Doutora em Enfermagem em Saúde Coletiva. Professora do Programa de Pós-Graduação em Atenção à Saúde da Universidade Federal do Triângulo Mineiro (UFTM), Uberaba, MG, Brasil.

Contato:

Helena Hemiko Iwamoto

Pós-Graduação em Enfermagem Universidade Federal do Triângulo Mineiro

Avenida Getúlio Guaritá, 107 - Uberaba, MG

CEP: 38025-440

E-mail:

helena.iwamoto@gmail.com

\section{Resumo}

Objetivo: caracterizar os acidentes de trabalho fatais e analisar a qualidade das informações de seus registros. Método: estudo ecológico com análise descritiva dos óbitos por acidentes de trabalho no Brasil, em Minas Gerais e no município de Uberaba, no período de 1997 a 2006. Utilizou-se como fonte de dados as informações do Sistema de Informações sobre Mortalidade (SIM), do Ministério da Saúde, e o Sistema Único de Benefícios (SUB), do Minis tério da Previdência e Assistência Social. Resultados: as causas mais comuns de acidentes de trabalho fatais estão relacionadas com os acidentes de trajeto e atingem principalmente homens da faixa etária entre 25 e 44 anos. Constatou-se um elevado percentual de informação ignorada no campo direcionado à identificação da relação do óbito com o acidente de trabalho, alcançando, no período, média de 82,9\% em nível nacional, de 84,5\% no estadual e de 79,6\% no municipal. Quanto à qualidade das informações do SIM, segundo os critérios propostos pela Comissão Econômica para a América Latina e o Caribe, é considerada muito ruim para informar sobre acidentes de trabalho, sendo um fator limitante para o planejamento de ações no campo da saúde do trabalhador a partir desta fonte de dados. Conclusão: são necessárias, portanto, formas mais eficazes de registro das informações relacionadas aos acidentes de trabalho fatais.

Palavras-chave: acidentes de trabalho; causas externas; causas de morte; notificação de acidentes de trabalho; saúde do trabalhador.

\begin{abstract}
Objective: The present study purpose is to characterize fatal occupational accidents and analyze the quality of recorded information. Method: Ecological study, with descriptive analysis of deaths due to occupational accidents in the city of Uberaba, the State of Minas Gerais, and Brazil, from 1997 to 2006. The study used data from the Information System on Mortality (SIM) of the Ministry of Health and from Workers' Benefit and Compensation System (SUB) from the Ministry of Social Security and Assistance. Results: The most common causes of work-related fatalities are commuting accidents and mainly involve males, aged between 25-44 years. The results showed that there is a high percentage of missing information in the records aimed at identifying the relationship between death and work accidents, reaching, during the period, the average of $82.9 \%$ in the national, $84.5 \%$ in the state and $79.6 \%$ in the municipal records. According to the criteria proposed by the Economic Commission for Latin America and Caribbean, the quality of information from the SIM is very poor, which results to be a limitation for action planning in the occupational health field based in such data. Conclusion: For this reason, it is necessary to adopt more effective ways of recording information related to occupational fatal accidents.
\end{abstract}

Keywords: occupational accidents; external causes; cause of death; occupational accidents registry; occupational health; fatal injuries.
Revisado: 02/05/2011

Aprovado: $12 / 05 / 2011$ 


\section{Introdução}

Acidentes de trabalho são fenômenos socialmente determinados e, em sua amplitude, previsível (WALDVOGEL, 2003). Esses acidentes afetam socioeconomicamente a realidade de um país, apresentando como decurso mais grave os casos fatais, resultando em perdas de anos potenciais de vida produtiva (SANTANA et al., 2007).

De acordo com relatórios da Organização Internacional do Trabalho (INTERNATIONAL LABOUR ORGANIZATION, 2003), estima-se a ocorrência de aproximadamente 270 milhões de acidentes de trabalho que resultam em 2 milhões de mortes por ano em todo o mundo. Estima-se, ainda, que até $4 \%$ do produto interno bruto de um país sejam gastos com problemas decorrentes de acidentes e doenças ocupacionais. Esses resultados caracterizam os acidentes de trabalho como importante problema de saúde pública mundial. Neste contexto, o Brasil ocupa o quarto lugar mundial em relação ao risco de morte no trabalho (GONÇALVES, 2007).

Vale destacar que a caracterização legal dos acidentes de trabalho ocorre:

pelo exercício do trabalho a serviço da empresa ou pelo
exercício do trabalho dos segurados especiais, provo-
cando, direta ou indiretamente, lesão corporal, doença
ou perturbação funcional que cause a morte ou a perda
ou redução, permanente ou temporária, da capacidade
para o trabalho. (BRASIL, 1991)

Podem ser classificados em duas categorias: aqueles que ocorrem no ambiente de trabalho e os que acontecem no trajeto para o local de trabalho (DIAS; CORDEIRO; GONÇALVES, 2006). Inclui-se nessa relação situações como os acidentes que ocorrem durante as viagens a serviço, mesmo que sejam realizadas com fins de estudo, mas desde que financiadas pela empresa (BRASIL, 2006; INSTITUTO DE PESQUISAS ECONÔMICAS APLICADAS, 2006).

Ressaltam-se as relações entre violência e processo de trabalho, uma vez que a violência decorrente de situações de trabalho é responsável por uma parcela significativa de casos de óbitos (MACHADO; GOMEZ, 1994; BRASIL, 2006; INSTITUTO DE PESQUISAS ECONÔMICAS APLICADAS, 2006). Situações nas quais os trabalhadores sofrem agressões, sabotagens ou atos de terrorismo praticados por colegas de trabalho ou terceiros, desde que estejam vinculados às atividades laborativas, são classificadas como acidentes de trabalho (BRASIL, 2006; INSTITUTO DE PESQUISAS ECONÔMICAS APLICADAS, 2006).

Com o intuito de reduzir a ocorrência dos acidentes de trabalho e das doenças ocupacionais, está em vigor no Brasil, desde 2004, a Política Nacional de Saúde do Trabalhador do Ministério da Saúde
(PNST/MS). Por meio de ações de promoção, reabilitação e vigilância na área da saúde, a PNST tem como atribuição identificar a relação entre ambiente, organização, condições de trabalho e os efeitos dessa teia de relações sobre a saúde do trabalhador (BRASIL, 2008).

Neste contexto, o principal instrumento para o reconhecimento dos acidentes de trabalho é a informação de suas ocorrências por meio da notificação (BRASIL, 2008). Através da notificação é possível identificar os motivos pelos quais os trabalhadores adoecem e morrem, pois se obtêm os dados relativos a número, tipo, horário, local de ocorrência de acidentes e características das vítimas, dentre outros (ROBAZZI et al., 2006; BRASIL, 2008). Cada um destes itens integra uma cadeia de fatos que devem ser considerados para a construção de medidas de prevenção e de controle dos acidentes (ROBAZZI et al., 2006).

Contudo, no Brasil, apesar da relevância dos acidentes de trabalho, no contexto das morbimortalidades em saúde, nem todos os casos chegam ao conhecimento das autoridades competentes, tanto pela falta de tradição dos profissionais de saúde em notificar, quanto pela omissão das empresas em cumprir a legislação vigente (MACHADO; GOMEZ, 1994; WALDVOGEL, 2002; WALDVOGEL, 2003; SANTANA; NOBRE; WALDVOGEL, 2005; SANTANA et al., 2007).

As informações que abrangem a mortalidade por acidentes de trabalho e seus aspectos epidemiológicos representam um fator crítico no sistema de saúde em relação à consolidação de uma base de dados completa e detalhada sobre esses casos em função das negligências na comunicação dos óbitos relacionados (SANTANA; NOBRE; WALDVOGEL, 2005; SANTANA et al., 2007).

$\mathrm{E}$, diante do entendimento de que a qualidade da informação gera conhecimento epidemiológico importante para o processo da organização das ações em saúde, o presente estudo tem por objetivo caracterizar os acidentes de trabalho fatais nos âmbitos nacional, estadual e municipal e analisar a qualidade dessas informações.

\section{Material e método}

Trata-se de estudo ecológico, descritivo e retrospectivo acerca das características dos óbitos por acidentes de trabalho e da qualidade dessas informações em três âmbitos - Brasil, Minas Gerais e Uberaba/MG - no período de 1996 a 2006. O objeto de análise foi o óbito por causas externas ao trabalhador vitimizado, identificado como acidente típico ou de trajeto. 
Na caracterização dos acidentes de trabalho fatais, foram analisados os dados do Sistema de Informações sobre Mortalidade (SIM), conforme se apresentam na tabulação disponível em página eletrônica do Departamento de Informática do Sistema Único de Saúde do Ministério da Saúde - Datasus/MS (BRASIL, 2009). Optou-se pela análise dos óbitos conforme classificação correspondente à $10^{\underline{a}}$ Revisão da Classificação Internacional de Doenças (CID-10), em vigor a partir de 1996, e representados por: acidentes de transporte (V01-V99); outras causas externas de lesões acidentais (W00-X59); lesões autoprovocadas voluntariamente (X60-X84); agressões (X85-Y09); eventos cuja intenção é indeterminada (Y10-Y34); e sequelas de causas externas (Y85-Y89) (ORGANIZAÇÃO MUNDIAL DA SAÚDE, 2000). As informações apresentadas no SIM têm como origem os dados das Declarações de Óbitos (DOs) de todo o território nacional. ${ }^{5}$ A caracterização procedeu-se conforme sexo, faixa etária e categoria CID-10.

Para análise da frequência de acidentes de trabalho fatais e da mortalidade no estado de Minas Gerais e no Brasil utilizaram-se os dados do Sistema Único de Benefícios (SUB) do Cadastro Nacional de Informações Sociais do Ministério da Previdência e Assistência Social - CNISMPAS (BRASIL, 2007).

O coeficiente de mortalidade para Brasil e Minas Gerais foi obtido dividindo-se o número de óbitos por acidentes de trabalho pela população sob risco de morrer por esses eventos por 100.000 trabalhadores segurados, considerando-se a base de dados provenientes do SUB/CNISMPAS.

Número de óbitos por acidentes de trabalho entre segurados com cobertura previdenciária

Número médio anual de segurados com cobertura previdenciária
A análise da qualidade das informações sobre os óbitos por acidentes de trabalho considerou o preenchimento do campo acidente de trabalho contido nas DOs registradas no SIM. A verificação da qualidade do preenchimento desta variável foi feita com base nos critérios propostos pela Comissão Econômica para a América Latina e o Caribe (Cepal), que considera a proporção de informação ignorada, os campos em branco e os códigos atribuídos como informação ignorada especificada, caracterizados como incompletude de informação. A Cepal considera excelente quando há menos de $5 \%$ de informações incompletas, bom de $5 \%$ a $10 \%$, regular de $10 \%$ a $20 \%$, ruim de $20 \%$ a $50 \%$ e muito ruim de $50 \%$ ou mais (ROMERO; CUNHA, 2006; OLIVEIRA et al., 2009).

Optou-se pela estatística descritiva por se tratar de um estudo descritivo e não ter a pretensão de teste de hipóteses. O presente estudo foi realizado em consonância com os preceitos da resolução CNS 196/96, não sendo considerado necessária a sua submissão a Comitê de Ética em Pesquisa, dada a natureza pública e administrativa das informações.

\section{Resultados}

Conforme dados do MPAS (Tabela 1), de 1997 a 2006, 11,67\% dos óbitos nacionais por acidentes de trabalho ocorreram em Minas Gerais, que apresentou no período um coeficiente médio de mortalidade maior que o nacional. Ressalta-se que as informações do MPAS referem-se exclusivamente aos trabalhadores contribuintes do Regime Geral de Benefícios da Previdência Social, disponíveis na página eletrônica somente a partir de $1997 .{ }^{6}$

Quanto à caracterização dos tipos de acidentes de trabalho fatais, na análise da distribuição temporal de

Tabela 1 Frequência e coeficiente de mortalidade por acidentes de trabalho fatais de trabalhadores beneficiários em Minas Gerais e Brasil de 1997 a 2006 segundo SUB/MPAS*

\begin{tabular}{|c|c|c|c|c|c|c|c|c|c|c|c|}
\hline Período & 1997 & 1998 & 1999 & 2000 & 2001 & 2002 & 2003 & 2004 & 2005 & 2006 & Total \\
\hline \multicolumn{12}{|l|}{ Frequência } \\
\hline Minas Gerais & 311 & 413 & 458 & 373 & 307 & 311 & 295 & 358 & 352 & 359 & 3537 \\
\hline Brasil & 2.819 & 3.793 & 3.896 & 3.094 & 2.753 & 2.968 & 2.674 & 2.839 & 2.766 & 2.717 & 30.319 \\
\hline \multicolumn{12}{|c|}{ Coeficiente de mortalidade (por 100.000 trabalhadores segurados) } \\
\hline Minas Gerais & 17,9 & 20,3 & 22,2 & 18,0 & 13,2 & 12,7 & 11,6 & 13,2 & 12,1 & 11,8 & 15,3 \\
\hline Brasil & 16,9 & 20,2 & 21,3 & 17,4 & 13,1 & 13,4 & 11,7 & 11,7 & 10,7 & 10,3 & 14,7 \\
\hline
\end{tabular}

*Fonte: SUB/MPAS - Sistema Único de Benefícios/Ministério da Previdência e Assistência Social. Disponível em: <www.previdenciasocial.gov.br>. Acesso em: 10 julho 2010.

\footnotetext{
${ }^{5}$ < http://tabnet.datasus.gov.br/cgi/deftohtm.exe?sim/cno/ext10mg.def>. Acesso em: 10 julho 2010.

${ }^{6}$ BRASIL. Ministério da Previdência e Assistência Social. Dataprev. Disponível em: <http://www3.dataprev.gov.br/infologo/GACT/ACT01/ACT01.php_>. Acesso em: 10 julho de 2010.
} 
1996 a 2006, conforme dados do SIM, observa-se predomínio dos acidentes de trajeto em relação às demais causas de acidentes de trabalho típicos, perfazendo assim uma das principais causas de mortalidade da classe trabalhadora (Tabela 2). Entretanto, apesar dos acidentes de trajeto serem a principal causa de morte, houve redução percentual desses óbitos entre os períodos de 1996 a 2000 e de 2001 a 2006, no Brasil, em Minas Gerais e em Uberaba (Tabela 2).

Em relação aos acidentes de trabalho típicos, a principal causa de óbito foi aquela relacionada a outras causas externas de lesões acidentais (W00-X59). Esse grupo engloba, de forma geral, as categorias: quedas, envenenamentos, sufocamentos e queimaduras (Tabela 2). Na comparação das porcentagens entre os períodos de 1996 a 2000 e de 2001 a 2006, observa-se aumento na ocorrência dos óbitos por acidentes de trabalho típicos no Brasil, em Minas Gerais e em Uberaba (Tabela 2).

No período entre 2001 e 2006, o MPAS registrou 1.982 casos de acidentes de trabalho fatais em Minas Gerais e 16.717 no Brasil (Tabela 1). Ao comparar esses dados com os do SIM (Tabela 2), observa-se uma divergência entre os dois registros, sendo maior o número de notificações do MPAS, totalizando uma diferença de 523 casos $(26,3 \%)$ para o estado de Minas Gerais e 27 casos $(0,2 \%)$ para o Brasil.

Na distribuição etária e por sexo dos óbitos decorrentes de acidentes de trabalho típicos e de trajeto, observa-se concentração no sexo masculino, com mais de 90\% dos óbitos relacionados, e adensamento nas faixas etárias produtivas, entre 25 e 44 anos. Além disso, os dados apontam para a ocorrência do trabalho infantil que, no contexto nacional, apresenta mortalidade em menores de cinco anos de $0,02 \%$ no período de 1996 a 2000 e aumento para $0,2 \%$ no período de 2001 a 2006 (Tabela 3).

Acerca da qualidade das informações originárias das DO (Tabela 4), observa-se elevado percentual de informações apresentadas como ignoradas no campo direcionado para a caracterização da relação do óbito com o acidente de trabalho. Durante o período de 1996 a 2006, a média de ausência desta informação (campo ignorado) foi de 82,9\% no âmbito nacional, de $84,5 \%$ no estado de Minas Gerais e de 79,6\% no município de Uberaba. Segundo os critérios definidos pelo Cepal para avaliar a qualidade do preenchimento dessa informação, esses resultados caracterizam como muito ruim, nas diferentes esferas, a capacidade das DOs retratarem as relações entre óbitos e acidentes de trabalho.

Tabela 2 Acidentes de trabalho fatais por grupo de causas no Brasil, em Minas Gerais e em Uberaba, de 1996 a 2006, segundo SIM*

\begin{tabular}{|c|c|c|c|c|c|c|c|c|c|c|c|c|}
\hline \multirow{3}{*}{$\begin{array}{c}\text { Acidentes de trabalho fatais } \\
\text { Grande Grupo } \\
\text { CID-10 }\end{array}$} & \multicolumn{4}{|c|}{ Brasil } & \multicolumn{4}{|c|}{ Minas Gerais } & \multicolumn{4}{|c|}{ Uberaba } \\
\hline & \multicolumn{2}{|c|}{1996 a 2000} & \multicolumn{2}{|c|}{2001 a 2006} & \multicolumn{2}{|c|}{1996 a 2000} & \multicolumn{2}{|c|}{2001 a 2006} & \multicolumn{2}{|c|}{1996 a 2000} & \multicolumn{2}{|c|}{2001 a 2006} \\
\hline & $N$ & $\%$ & N & $\%$ & $N$ & $\%$ & $N$ & $\%$ & $N$ & $\%$ & $N$ & $\%$ \\
\hline \multicolumn{13}{|l|}{ De trajeto } \\
\hline $\begin{array}{l}\text { Acidente de transporte } \\
\text { (V01-V99) }\end{array}$ & 5.630 & 49,7 & 7.789 & 46,7 & 347 & 55,3 & 770 & 52,7 & 19 & 65,5 & 31 & 59,6 \\
\hline \multicolumn{13}{|l|}{ Típico } \\
\hline $\begin{array}{l}\text { Outras causas externas } \\
\text { de lesões acidentais } \\
\text { (WOO-X59) }\end{array}$ & 5.681 & 50,1 & 8.717 & 52,2 & 280 & 44,7 & 681 & 46,6 & 10 & 34,5 & 21 & 40,4 \\
\hline 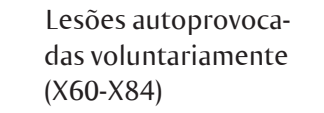 & 1 & 0,0 & 10 & 0,1 & 0 & 0,0 & 0 & 0,0 & 0 & 0,0 & 0 & 0,0 \\
\hline Agressões (X85-Y09) & 7 & 0,1 & 114 & 0,7 & 0 & 0,0 & 0 & 0,0 & 0 & 0,0 & 0 & 0,0 \\
\hline $\begin{array}{l}\text { Eventos de intenção in- } \\
\text { determinada (Y10-Y34) }\end{array}$ & 7 & 0,1 & 49 & 0,3 & 0 & 0,0 & 7 & 0,5 & 0 & 0,0 & 0 & 0,0 \\
\hline $\begin{array}{l}\text { Sequelas de causas } \\
\text { externas (Y85-Y89) }\end{array}$ & 3 & 0,0 & 11 & 0,1 & 0 & 0,0 & 2 & 0,0 & 0 & 0,0 & 0 & 0,0 \\
\hline Total & 11.329 & 100 & 16.690 & 100 & 627 & 100 & 1.460 & 100 & 29 & 100 & 52 & 100 \\
\hline
\end{tabular}

*Fonte: Sistema de Informações sobre Mortalidade (SIM), Departamento de Informática do Sistema Único de Saúde do Ministério da Saúde. Disponível em: <www.datasus.gov.br>. Acesso em: 10 out. 2009. 
Tabela 3 Acidentes de trabalho fatais por sexo e faixa etária no Brasil, Minas Gerais e Uberaba, de 1996 a 2006, segundo SIM*

\begin{tabular}{|c|c|c|c|c|c|c|c|c|c|c|c|c|}
\hline \multirow{3}{*}{$\begin{array}{c}\text { Acidentes de } \\
\text { trabalho } \\
\text { fatais }\end{array}$} & \multicolumn{4}{|c|}{ Brasil } & \multicolumn{4}{|c|}{ Minas Gerais } & \multicolumn{4}{|c|}{ Uberaba } \\
\hline & \multicolumn{2}{|c|}{1996 a 2000} & \multicolumn{2}{|c|}{2001 a 2006} & \multicolumn{2}{|c|}{1996 a 2000} & \multicolumn{2}{|c|}{2001 a 2006} & \multicolumn{2}{|c|}{1996 a 2000} & \multicolumn{2}{|c|}{2001 a 2006} \\
\hline & $N$ & $\%$ & $N$ & $\%$ & $N$ & $\%$ & $N$ & $\%$ & $N$ & $\%$ & $N$ & $\%$ \\
\hline \multicolumn{13}{|l|}{ Sexo } \\
\hline Masculino & 10.784 & 95,2 & 16.026 & 96,0 & 591 & 94,3 & 1.401 & 96,0 & 26 & 89,7 & 49 & 94,2 \\
\hline Feminino & 538 & 4,7 & 662 & 4,0 & 32 & 5,1 & 59 & 4,0 & 3 & 10,3 & 3 & 5,8 \\
\hline Sem registro & 7 & 0,1 & 2 & 0,0 & 4 & 0,6 & 0 & 0,0 & 0 & 0,0 & 0 & 0,0 \\
\hline \multicolumn{13}{|l|}{ Faixa etária } \\
\hline$<5$ & 2 & 0,02 & 5 & 0,2 & 0 & 0,0 & 0 & 0,0 & 0 & 0,0 & 0 & 0,0 \\
\hline $5-14$ & 134 & 1,2 & 102 & 0,6 & 5 & 0,8 & 7 & 0,5 & 1 & 3,4 & 0 & 0,0 \\
\hline $15-24$ & 2.151 & 19,0 & 2.764 & 14,6 & 118 & 18,8 & 212 & 14,5 & 3 & 10,3 & 6 & 11,5 \\
\hline $25-34$ & 3.190 & 28,2 & 4.393 & 25,4 & 155 & 24,7 & 365 & 25,0 & 6 & 20,7 & 11 & 21,2 \\
\hline $35-44$ & 2.902 & 25,6 & 4.246 & 25,2 & 174 & 27,8 & 387 & 26,5 & 5 & 17,2 & 15 & 28,8 \\
\hline $45-54$ & 1.721 & 15,2 & 3.075 & 19,8 & 101 & 16,1 & 309 & 21,2 & 4 & 13,8 & 15 & 28,8 \\
\hline $55-64$ & 851 & 7,5 & 1.417 & 10,0 & 55 & 8,8 & 117 & 8,0 & 5 & 17,2 & 4 & 7,7 \\
\hline$>65$ & 339 & 3,0 & 661 & 4,0 & 16 & 2,6 & 62 & 4,2 & 4 & 13,8 & 1 & 1,9 \\
\hline Ignorada & 39 & 0,3 & 27 & 0,3 & 3 & 0,5 & 1 & 0,1 & 1 & 3,4 & 0 & 0,0 \\
\hline Total & 11.329 & 100 & 16.690 & 100 & 627 & 100 & 1.460 & 100 & 29 & 100 & 52 & 100 \\
\hline
\end{tabular}

* Fonte: Sistema de Informações sobre Mortalidade (SIM), Departamento de Informática do Sistema Único de Saúde do Ministério da Saúde. Disponível em: <www.datasus.gov.br>. Acesso em: 10 out. 2009.

Tabela 4 Registros das declarações de óbitos por causas externas segundo informações relacionadas com acidentes de trabalho fatais (ATF), não relacionadas com ATF e com informação ignorado/em branco para ATF, no Brasil, em Minas Gerais e em Uberaba de 1996 a 2006

\begin{tabular}{|c|c|c|c|c|c|c|c|c|}
\hline \multirow{2}{*}{ Período } & \multicolumn{2}{|c|}{ Relacionadas com ATF } & \multicolumn{2}{|c|}{$\begin{array}{c}\text { Não } \\
\text { relacionadas com ATF }\end{array}$} & \multicolumn{2}{|c|}{$\begin{array}{c}\text { Com informação ignora- } \\
\text { do/branco para ATF }\end{array}$} & \multicolumn{2}{|c|}{ Total } \\
\hline & $N$ & $\%$ & $N$ & $\%$ & N & $\%$ & N & $\%$ \\
\hline \multicolumn{9}{|l|}{ Brasil } \\
\hline 1996 a 2000 & 11.329 & 1,9 & 67.071 & 11,3 & 513.287 & 86,7 & 591.687 & 100 \\
\hline 2001 a 2006 & 16.690 & 2,2 & 141.518 & 18,7 & 599.444 & 79,1 & 757.652 & 100 \\
\hline \multicolumn{9}{|l|}{ Minas Gerais } \\
\hline 1996 a 2000 & 627 & 1,3 & 3.054 & 6,5 & 43.311 & 92,2 & 46.992 & 100 \\
\hline 2001 a 2006 & 1.460 & 2,3 & 12.934 & 20,0 & 50.185 & 77,7 & 64.579 & 100 \\
\hline \multicolumn{9}{|l|}{ Uberaba } \\
\hline 1996 a 2000 & 29 & 3,3 & 85 & 9,8 & 756 & 86,9 & 870 & 100 \\
\hline 2001 a 2006 & 52 & 5,6 & 205 & 22,1 & 672 & 72,1 & 929 & 100 \\
\hline
\end{tabular}

Fonte: Sistema de Informações sobre Mortalidade (SIM), Departamento de Informática do Sistema Único de Saúde do Ministério da Saúde. Disponível em: <www.datasus.gov.br>. Acesso em: 10 out. 2009. 


\section{Discussão}

Os coeficientes nacional e de Minas Gerais apresentam-se, aproximadamente, 20 vezes maiores que o estimado em países desenvolvidos, como a Inglaterra, onde a mortalidade por acidentes de trabalho é de 0,7/100.000 (SANTANA et al., 2007). Ressalta-se que a análise do coeficiente de mortalidade expressa a magnitude dos óbitos por acidente de trabalho, além de evidenciar a fragilidade dos níveis de segurança proporcionados nos ambientes de trabalho (SANTANA; NOBRE; WALDVOGEL, 2005; SANTANA et al., 2007).

Em relação às características demográficas, a mortalidade por acidentes de trabalho concentra-se no sexo masculino e nas faixas etárias dos adultos jovens, resultando em perda de vida produtiva social e econômica. Esse perfil condiz com o panorama nacional (BRASIL, 2006; INSTITUTO DE PESQUISAS ECONÔMICAS APLICADAS, 2006). O estudo de Santana et al. (2007) acerca dos Anos Potenciais de Vida Perdidos (APVP) em decorrência dos acidentes de trabalho, no estado da Bahia, também demonstra que trabalhadores nas faixas etárias de 20 a 25 anos e 35 a 39 anos apresentam maior impacto na produtividade.

Os resultados aqui obtidos também refletem a composição contemporânea do mercado de trabalho, tendo em vista a maior presença do sexo masculino no mercado produtor em relação ao sexo feminino (BRASIL, 2006; INSTITUTO DE PESQUISAS ECONÔMICAS APLICADAS, 2006). Com isso, os homens adultos, que detêm maior força de trabalho produtiva, apresentam-se como grupo de maior vulnerabilidade para os acidentes de trabalho fatais (BARATA; RIBEIRO; MORAES, 2000; WALDVOGEL, 2003; BRASIL, 2006; ROBAZZI et al., 2006).

Em 1996, o Brasil implantou o Programa de Erradicação do Trabalho Infantil (Peti), para combater as formas de trabalho consideradas mais perigosas, insalubres, degradantes, assim como a inserção precoce no mundo do trabalho (CARVALHO, 2004). Entretanto, casos de morte por acidente de trabalho de menores de idade registrados no território nacional no período estudado, inclusive para menores de 14 anos e até em menores de 5 anos, indicam a gravidade dessa situação. Esses resultados exigem o fortalecimento das estratégias de intervenção.

Estudos relacionados aos tipos de acidentes de trabalho fatais apresentam aumento da prevalência de acidentes de trajeto em relação aos acidentes típicos, principalmente a partir da década de 1990. Esse fato aponta para a interação entre fenômenos da violência urbana e a progressiva exposição dos trabalhadores aos acidentes de trânsito fatais (MACHADO; GOMEZ, 1994; HENNINGTON; CORDEIRO; MOREIRA FILHO, 2004).

Em relação à qualidade das informações, a fonte de dados sob a responsabilidade do MPAS circuns- creve-se à divulgação eletrônica de registros básicos como categoria profissional, tipo de acidente e setor de trabalho (HENNINGTON; MONTEIRO, 2006). As análises decorrentes dessas informações limitam a elaboração de um diagnóstico completo da situação de saúde do trabalhador, uma vez que não abrangem informações a respeito da morbimortalidade referente ao trabalhador informal ou aos demais trabalhadores, como servidores públicos e militares. Essa informação está condicionada à declaração de interesse do segurado para a concessão do benefício previdenciário através da Comunicação de Acidente de Trabalho - CAT (BARATA; RIBEIRO; MORAES, 2000; HENNINGTON; CORDEIRO; MOREIRA FILHO, 2004).

Por outro lado, o SIM é desenvolvido pelo Departamento de Informática do Sistema Único de Saúde (Datasus) e tem a importância de consolidar todas as DOs emitidas pelos serviços de saúde e por cartórios de registro civil. A DO é considerada uma fonte ágil para enumerar e quantificar os acidentes relacionados ao trabalho, identificar riscos e subsidiar os serviços que trabalham com vigilância em saúde do trabalhador (BRASIL, 2008). Entretanto, apesar da dimensão universal dos registros de acidentes de trabalho nas DOs, que não dependem das relações empregatícias do cidadão para sua definição, é evidenciada maior incompletude na qualidade dos registros dessas informações quando comparadas ao sistema da Previdência Social (MACHADO; GOMES, 1994; SANTANA; NOBRE; WALDVOGEL, 2005; WALDVOGEL, 2002; ROMERO; CUNHA, 2006; SANTANA et al., 2007).

A qualidade dos registros das DOs disponibilizadas pelo SIM/Datasus, em termos de notificação de acidentes de trabalho fatais, segundo os critérios propostos pela Cepal, pode ser considerada muito ruim, uma vez que é elevado o percentual de informações apresentadas como ignoradas com cerca de $80 \%$ nas três esferas estudadas. A realidade de incompletude das informações se repete em diferentes localidades nacionais. Oliveira e Mendes (1997) demonstraram que apenas duas dentre as 31 DOs investigados em Porto Alegre apresentaram campo preenchido relacionado ao acidente de trabalho. Carneiro (2000) identificou 67,7\% de sub-registro nas DOs, quando comparadas aos Boletins de Ocorrência das delegacias de Polícia da Zona Norte de São Paulo, sobre acidentes de trabalho fatais. Correa e Assunção (2003) encontraram incoerência na relação entre os óbitos registrados no Sistema de Informação de Acidentes de Trabalho do SUS de Belo Horizonte e por ausência de preenchimento adequado de campo referente aos acidentes de trabalho em 98\% das DOs. Santana, Nobre e Waldvogel (2005) apresentaram, em suas comparações entre os dados das DOs e dos registros da Previdência Social, uma estimativa de sub-registro em $56,8 \%$ no país.

O preenchimento deficitário dos campos das DOs compromete a qualidade e a pertinência das informa- 
ções disponíveis, transformando-se em obstáculo para a adoção adequada da vigilância em saúde do trabalhador (MACHADO; GOMEZ, 1994; BRASIL, 2008). Com isso, a violência decorrente das situações de trabalho, responsável por uma parcela significativa de óbitos, que está representada na classificação internacional da mortalidade por causas externas, não é evidenciada em toda a sua magnitude (MACHADO; GOMEZ, 1994; BRASIL, 2008).

Nesse sentido, as implicações relacionadas à incompletude das informações são complexas no cenário da saúde atual, quer seja pela influência nos serviços de saúde disponíveis ou na definição de prioridades governamentais (WALDVOGEL, 2002; CORREA; ASSUNÇÃO, 2003; OLIVEIRA et al., 2009).

Entre os fatores referentes à manutenção dessa lacuna na abrangência e na qualidade dos dados, destaca-se o fato dos médicos preencherem os formulários das DOs apenas para cumprir uma formalidade, sem preocupação com o valor dessas informações (CARNEIRO, 2000; WALDVOGEL, 2002; OLIVEIRA et al., 2009). De modo geral, os médicos, ao preencherem as DOs, demonstram maior preocupação em informar a causa imediatamente associada ao óbito e não os outros fatores determinantes. Neste contexto, é fundamental a sensibilização destes profissionais para a completude dos registros como forma de contribuição para a organização efetiva de políticas de saúde voltados ao trabalhador (BARATA; RIBEIRO; MORAES, 2000).

Salienta-se, nesta discussão, o caráter jurídico e civil das DOs e sua definição como instrumento oficial, considerada de suma importância para a quantificação de agravos fatais na população brasileira (CORREA; ASSUNÇÃO, 2003). Além disso, a dificuldade de obtenção de dados sobre morbimortalidade dos trabalhadores pode estar relacionada à falta de integração entre os diversos Sistemas de Informação em Saúde (SIS) ou até mesmo à carência de um sistema efetivo para a captação destas informações (CARNEIRO, 2000; CORREA; ASSUNÇÃO, 2003; BRASIL, 2006; INSTITUTO DE PESQUISAS ECONÔMICAS APLICADAS, 2006).

A continuidade da baixa qualidade das informações registradas sobre os acidentes de trabalho fatais, ao longo do período analisado, evidencia a necessidade de se realizar investigações sobre as razões que contribuem para: o erro de registro, a falta de associação do óbito com o trabalho da víti- ma e a incoerência entre o registro do acidente ou a violência que ocasionou a lesão fatal em relação à causa básica do óbito (CARNEIRO, 2000; CORREA; ASSUNÇÃO, 2003; OLIVEIRA et al., 2009).

\section{Conclusão}

As características dos acidentes de trabalho fatais, para o período de 1996 a 2006, apresentam maior prevalência dos acidentes de trajeto em relação aos acidentes típicos. Entretanto, há uma redução da percentagem dos acidentes de trajeto ao se comparar os períodos de 1996 a 2000 e de 2001 a 2006, nas três esferas analisadas: Brasil, Minas Gerais e o município de Uberaba.

Para os acidentes de trabalho típicos, as principais causas de morte foram aquelas incluídas no grupo de outras causas externas de lesões acidentais (W00-X59). Os homens na fase adulta constituiram o maior percentual de vítimas, fato que destaca o caráter limitador da vida produtiva, econômica e social que os acidentes de trabalho fatais impõem.

Em torno de 80\% das DOs registradas no Brasil, em Minas Gerais e no município de Uberaba, de 1996 a 2006, apresentaram o campo referente aos acidentes de trabalho ignorado, portanto, considera-se a qualidade das informações acerca dos acidentes de trabalho muito ruim. Ressalta-se que essa lacuna nas informações dificulta o diagnóstico sobre o real problema que envolve os acidentes de trabalhos fatais, sendo considerada como fator limitante para o planejamento de ações no campo da saúde do trabalhador.

Neste contexto, a notificação integral dos acidentes de trabalho fatais e o seu efetivo controle permanecem no cenário da saúde como um desafio. Por isso, é urgente buscar formas mais eficazes para que haja a valorização do preenchimento dessa informação nas DOs, como integração entre os diferentes bancos de dados, em busca de captação mais precisa das notificações dos óbitos relacionados ao trabalho.

Ao contrário do que o nome sugere, os acidentes de trabalho não são eventos fortuitos ou acidentais, mas fenômenos socialmente previsíveis e preveníveis. Por essas razões, a fim de se propor enfrentamento efetivo e promoção da saúde do trabalhador, há necessidade de que os acidentes de trabalho fatais sejam revelados com mais precisão através da melhoria da qualidade das informações em saúde.

\section{Contribuições de autoria}

Iwamoto, H. H.: coordenou a pesquisa e participou da revisão da versão final do manuscrito. Camargo, F. C.: realizou o delineamento do manuscrito, a análise dos dados e participou da elaboração e da versão final do manuscrito. Tavares, L. C.: participou da elaboração do manuscrito e da coleta dos dados. Miranzi, S. S. C.: participou da análise dos dados e da revisão do manuscrito. 


\section{Referências}

BARATA, R. C. B.; RIBEIRO, M. C. S. A.; MORAES, J. C. Acidentes de trabalho referidos por trabalhadores moradores em área urbana no interior de São Paulo em 1994. Informe Epidemiológico do SUS, Brasília, v. 9, n. 3, p. 199-210, 2000.

BRASIL. Lei $n^{\circ}$ 8.213, de 24 de julho de 1991. Dispõe sobre planos de benefícios da previdência social e dá outras providências. Diário Oficial [da] República Federativa do Brasil, Brasília, DF, 14 ago. 1991. Disponível em: <http:/www010.dataprev.gov.br/sislex/ paginas/42/1991/8213.HTM>. Acesso em: 20 out. 2011.

. Ministério da Previdência e Assistência Social. Anuário estatístico da previdência social 2007. Disponível em: <http://www.previdenciasocial.gov.br/estatisticas >. Acesso em: 10 out. 2009.

. Ministério da Saúde. Departamento de Informática do Sistema Único de Saúde (Datasus). Brasília, [s.d.]. Disponível em: <http:// www.datasus.gov. br>. Acesso em: 10 out. 2009.

. Ministério da Saúde. Secretaria de Atenção à Saúde. Departamento de Ações Programáticas Estratégicas. Notificação de acidentes do trabalho fatais, graves e com crianças e adolescentes. Brasília, DF, 2006.

. Ministério da Saúde. Secretaria de Vigilância em Saúde. Sobre a Saúde do Trabalhador. Brasília, 2008. Disponível em: <http://portal.saude.gov.br/portal/ saude $>$. Acesso em: 16 jun. 2009.

CARNEIRO, S. A. M. Trabalho e violência: relação de proximidade da violência a trabalhadores durante a jornada de trabalho, na Zona Norte de São Paulo em 1998. 149f. Dissertação (Mestrado em Saúde Pública)Faculdade de Saúde Pública, Universidade de São Paulo, São Paulo, 2000.

CARVALHO, I. M. M. Algumas lições do Programa de Erradicação do Trabalho Infantil. São Paulo em Perspectiva, São Paulo, v. 18, n. 4, p. 50-61, out./dez. 2004.

CORREA, P. R. L.; ASSUNÇÃO, A. A. A subnotificação de mortes por acidentes de trabalho: estudo de três bancos de dados. Epidemiologia e Serviços de Saúde, Brasília, v. 12, n. 4, p. 203-12, 2003.

DIAS, A.; CORDEIRO, R.; GONÇALVES, C. G. O. Exposição ocupacional ao ruído e acidentes do trabalho. Cadernos de Saúde Pública, Rio de Janeiro, v. 22, n. 10, p. 2125-30, out. 2006.

GONÇALVES, J. A. Acidente de trabalho entre a equipe assistencial multiprofissional: uma avaliação da subnotificação. 102f. Dissertação (Mestrado em Enfermagem)-Escola de Enfermagem, Universidade Federal de Minas Gerais, Belo Horizonte, 2007.

HENNINGTON, E. A.; CORDEIRO, R.; MOREIRA FILHO, D. C. Trabalho, violência e morte em Campinas, São
Paulo, Brasil. Cadernos de Saúde Pública, Rio de Janeiro, v. 20, n. 2, p. 610-617, 2004.

HENNINGTON, E. A.; MONTEIRO, M. O perfil epidemiológico dos acidentes de trabalho no Vale dos Sinos e o sistema de vigilância em saúde do trabalhador. História, Ciências, Saúde-Manguinhos, Rio de Janeiro, v. 13, n. 4, p. 865-76, out./dez. 2006

INTERNATIONAL LABOUR ORGANIZATION. Safety in numbers: pointers for the global safety culture at work. Geneva, 2003.

INSTITUTO DE PESQUISAS ECONÔMICAS

APLICADAS. Brasil: o estado de uma nação: mercado de trabalho, emprego e informalidade. Brasília, DF, 2006.

MACHADO, J. M. H.; GOMEZ, C. M. Acidentes de trabalho: uma expressão da violência social. Cadernos de Saúde Pública, Rio de Janeiro, v. 10, p. 74-87, 1994. Suplemento 1.

OLIVEIRA, M. E. P. et al. Avaliação da completitude dos registros de febre tifóide notificados no Sinan pela Bahia. Epidemiologia e Serviços de Saúde, Brasília, v. 18, n. 3, p. 219-226, jul./set. 2009.

OLIVEIRA, P. A. B.; MENDES, J. M. Acidentes de trabalho: violência urbana e morte em Porto Alegre, Rio Grande do Sul, Brasil. Cadernos de Saúde Pública, Rio de Janeiro, v. 13, p. 73-83, 1997. Suplemento 2.

ORGANIZAÇÃO MUNDIAL DA SAÚDE. Classificação estatística internacional de doenças e problemas relacionados à saúde: CID-10. 10 $0^{\underline{a}}$ revisão. 8.ed. São Paulo: EdUsp, 2000.

ROBAZZI, M. L. C. C. et al. O prontuário hospitalar auxiliando na identificação da violência no trabalho. Revista de Enfermagem UERJ, Rio de Janeiro, v. 14, n. 4, p. 499-505, out./dez. 2006.

ROMERO, D. E.; CUNHA, C. B. Avaliação da qualidade das variáveis sócio-econômicas e demográficas dos óbitos de crianças menores de um ano registrados no Sistema de Informações sobre Mortalidade do Brasil (1996/2001). Cadernos de Saúde Pública, Rio de Janeiro, v. 22, n. 3, p. 673-684, mar. 2006.

SANTANA, V. S.; NOBRE, L.; WALDVOGEL, B. C. Acidentes de trabalho no Brasil entre 1994 e 2004: uma revisão. Ciência e Saúde Coletiva, Rio de Janeiro, v. 10, n. 4, p. 841-855, 2005.

SANTANA, V. S. et al. Mortalidade, anos potenciais de vida perdidos e incidência de acidentes de trabalho na Bahia, Brasil. Cadernos de Saúde Pública, Rio de Janeiro, v. 23, n. 11, p. 2643-2652, nov. 2007.

WALDVOGEL, B. C. Acidentes do trabalho: os casos fatais - a questão da identificação e da mensuração. Belo Horizonte: Segrac, 2002.

A população trabalhadora paulista e os acidentes do trabalho fatais. São Paulo em Perspectiva, São Paulo, v. 7, n. 2, p. 42-53, 2003. 